\title{
Correspondence
}

\section{An Undergraduate Study for the Comparison of Dissolution Profiles using 3D Printed PVA and Commercial Paracetamol Tablets}

\author{
Maryam Asmari, Bruno C. Sil, Bhaven Patel ${ }^{*}$ \\ School of Human Sciences, London Metropolitan University, 166-220 Holloway Road, London N7 8DB, UK
}

\author{
ARTICLE INFO \\ Article History \\ Received 04 May 2020 \\ Accepted 27 August 2020 \\ Keywords \\ $3 \mathrm{D}$ printing \\ polyvinyl alcohol \\ paracetamol tablets \\ fused deposition modelling \\ sustained release
}

(C) 2020 The Authors. Published by Atlantis Press B.V. This is an open access article distributed under the CC BY-NC 4.0 license (http://creativecommons.org/licenses/by-nc/4.0/).

\section{INTRODUCTION}

Three-dimensional Printing (3DP) is an additive manufacturing process that can rapidly render $3 \mathrm{D}$ objects from computer aided design software by successively depositing polymeric materials layer by layer [1]. A range of different 3DP technologies are in current use, with the most prevalent methodology using Fused Deposition Modelling (FDM) technology due to a synergetic combination that merges economic and accuracy/ resolution factors [2]. In 2015, Spritam;, a solid pharmaceutical formulation which contains the anti-epileptic drug levetiracetam, became the first $3 \mathrm{D}$ printed medicine approved by the US Food and Drug Administration leading to a raised interest in this type of technology so to revolutionise healthcare in the area of personalised medicines [3].

Paracetamol, a Class III Biopharmaceutics Classification System drug (low permeability, high solubility), is a well-known analgesic and antipyretic drug that currently available in different pharmaceutical dosage forms including tablets, suspensions and solutions. Tablets are recurrently the most common accessible paracetamol containing formulation and are typically produced using the direct powder compression methodology or granulation, if segregation of powder constituents is a problem $[4,5]$. However, these manufacturing processes exhibit limitations when compared to the extrusion method of 3DP. The most imperative limitation of the granulation method is lower Active Pharmaceutical Ingredient (API) loading resulting in higher final product total weight and material loss during processing [6], making 3DP an appealing alternative method for tableting manufacturing.
Few studies have investigated the production of paracetamol formulations using FDM printing [7,8]. Numerous studies have loaded drugs onto polymer filaments. Polyvinyl Alcohol (PVA), frequently utilised in coatings for tablets, is a commonly used filament and impregnation of the drug is achieved by soaking the filament in a highly saturated drug solution. This method results in low drug loading $(<2 \% \mathrm{w} / \mathrm{w})$ because of slow drug diffusion into polymer $[9,10]$. The use of hot melt extrusion has been reviewed as an alternative method by Tan to increase drug loading of API onto filaments [11]. However, to date there are no direct comparisons of the release profiles of 3D printed paracetamol tablets and commercial tablets. PVA is one of the most widely used water-soluble synthetic polymers and was selected because of its excellent thermoplasticity and it is the only commercially extruded polymer filament that would dissolve in vivo $[9,12]$. Herein we demonstrate the preparation of $3 \mathrm{D}$ printed paracetamol tablets using PVA, a biocompatible polymeric construct, and compare the drug release dissolution profile of the API with commercially available paracetamol containing tablets.

\section{MATERIALS AND METHODS}

\subsection{Materials}

Paracetamol USP grade, methanol (for HPLC, $\geq 99.9 \%$ ) and potassium phosphate monobasic $\left(\mathrm{KH}_{2} \mathrm{PO}_{4}\right)$ were purchased from SigmaAldrich Ltd, Gillingham, UK. PVA was purchased from 3D FilaPrint, Southend-on-Sea, UK. Milli-Q water (resistivity $18.2 \mathrm{M} \Omega \mathrm{cm}$ ) was used to produce all solutions. Paracetamol tablets were purchased from a pharmaceutical retailer (Boots, Nottingham, UK) with the following specifications: Paracetamol $500 \mathrm{mg}$ Tablets (Batch No. 109T, Exp. Date. 08/2020, M\&A Pharmachem Ltd, Bolton, UK). 


\subsection{Methods}

\subsubsection{Preparation of paracetamol-loaded PVA filament}

Polyvinyl alcohol filament was cut into small pieces and milled using a Krups F20342 grinder (KRUPS, Windsor, UK) and sieved through a $1000 \mu \mathrm{m}$ mesh. The milled PVA (100 g) and paracetamol powder $(100 \mathrm{~g})$ were combined using a mortar and pestle until a homogenous mixture was produced. The mixture was then extruded using a single-screw filament extruder (Wellzoom desktop extruder filament B, Shanghai, China) to obtain a drug loaded filament (extrusion temperature: $200{ }^{\circ} \mathrm{C}$; nozzle diameter: $3 \mathrm{~mm}$; screw speed: $25 \mathrm{rpm})$. The extruded filament was stored in a vacuum desiccator (Nalgene, Rochester, USA) and protected from light until printing. The drug-loading of the filament was determined by UV analysis.

\subsubsection{Additive manufactured paracetamol- loaded tablets printing specifications}

Tablets were fabricated using the aforementioned PVA drug-loaded filaments and a commercial fused-deposition modelling 3D printer, Ultimaker 2 (Ultimaker, Geldermalsen, Netherlands). Tablets were designed using the freeware web-based application Tinkercad (Autodesk, San Rafael, USA) software, exported as a Standard Tessellation Language file and uploaded to the 3D printer software, Cura (Ultimaker). The 3D geometry of the tablet was cylindrical (12.83 $\mathrm{mm}$ diameter $\times 4.10 \mathrm{~mm}$ height). The fill density was set at $150 \%$ in order to produce solid dosage forms of high density. The printer settings that were found to produce the best tablets for PVA drug-loaded filament were of fine resolution, nozzle temperature $\left(200{ }^{\circ} \mathrm{C}\right)$, nozzle diameter $(0.4 \mathrm{~mm})$, speed while extruding $(30 \mathrm{~mm} / \mathrm{s})$, speed while travelling $(30 \mathrm{~mm} / \mathrm{s})$, material flow $(150 \%)$, number of shells (2), layer height $(0.10 \mathrm{~mm})$ and build plate temperature $\left(60^{\circ} \mathrm{C}\right)$. No supports or rafts were utilised in the printed model.

\subsubsection{UV analysis and quantification of paracetamol samples}

Paracetamol analysis was conducted using a Genova Plus UV/ Visible spectrophotometer (Cole-Parmer, Staffordshire, UK). The UV readings were acquired at a wavelength of $242 \mathrm{~nm}$. A stock solution $(24 \mu \mathrm{g} / \mathrm{mL})$ was prepared from a known amount of paracetamol dissolved in a $2 \%$ methanolic solution. The stock solution was diluted accordingly to prepare various concentrations of paracetamol ranging from 1.2 to $24 \mu \mathrm{g} / \mathrm{mL}$. A calibration curve was constructed with a linear relationship between concentration and absorbance with regression coefficient values $\left(r^{2}\right)$ of greater than 0.999 .

\subsubsection{Testing conditions for tablet dissolution}

Tablet dissolution profiles for both commercially available and 3D printed formulations $(n=3)$ were obtained using a USP II apparatus (Caleva 10ST, Erweka $\mathrm{GmbH}$, Germany). The formulations were placed in $900 \mathrm{~mL}$ of a $\mathrm{KH}_{2} \mathrm{PO}_{4}$ buffer solution ( $\mathrm{pH} 5.8,0.032 \mathrm{M}$ ) for $60 \mathrm{~min}$ to simulate the primary environment of the small intestine compartment. Paddle speed for USP II equipment was fixed at
$50 \mathrm{rpm}$ and the sampling conducted at $37 \pm 0.5^{\circ} \mathrm{C}$ under sink conditions. Aliquots from the dissolution media $(1 \mathrm{~mL})$ were taken at standardised time intervals and the volume replaced with fresh $\mathrm{KH}_{2} \mathrm{PO}_{4}$ buffer solution ( $\mathrm{pH} 5.8,0.032 \mathrm{M})$. Dissolution media samples were diluted to fit the calibration curve and paracetamol concentration was determined using the aforementioned UV analysis methodology.

\section{RESULTS AND DISCUSSION}

Herein we show how PVA was used for the printing of tablets as a main polymeric filler. PVA is a well-known excipient used in the pharmaceutical industry acting as a binder in solid drug formulation [13]. This biocompatible polymer presents various desirable qualities for medicine manufacture such as high-water solubility and tensile modulus (3500 MPa) [14]. Unlike polycaprolactone, another biocompatible polymer with lower water solubility and extrusion temperature, PVA was shown to have positive FDM 3DP capabilities which intend to recreate other important tablet properties [i.e. hardness, friability, disintegration (shown in the Supplementary Materials) and dissolution] [14].

Paracetamol loaded PVA filament $(3 \mathrm{~mm}$ ) was successfully manufactured by blending a mixture of the API and PVA using hot melt extrusion. Tablets were initially fabricated using neat PVA filament to simulate the optimum conditions for 3DP (Figure 1A). Drug loaded printlets were similarly produced in cylindrical shape (Figure 1B). The drug loading of the filament was 39\%. This was lower than the theoretical value as a result of adhesion of the fine API powder to the walls of the barrel of the HME during the extrusion process [15]. The tablets showed similar values to the filament indicating that no degradation took place during the printing process. Tablets also showed high uniformity in size and weight (mean dimensions: $12.93 \mathrm{~mm}$ diameter $\times 4.08 \mathrm{~mm}$ height, mean weight: $1.2459 \mathrm{~g}$ ) which rendered a concise method of manufacture. Erokhin et al. [16] have demonstrated that higher infill densities of $3 \mathrm{D}$ scaffolds reduce the shrinkage of polymers when printing thus resulting in marginally larger diameters in comparison to the original designs. The height of the 3D printed construct shown here was comparable to the designed tablet, also demonstrating good adhesion between the printed layers. The dose of paracetamol in the 3D printed tablet was $484.6 \pm 18 \mathrm{mg}$ in comparison to the commercial tablets that contained $500 \pm 2 \mathrm{mg}$.

The dissolution profiles of commercial and PVA-loaded 3D printed paracetamol tablets were collected over a period of $60 \mathrm{~min}$ as shown in Figure 2. The results gathered for the dissolution of the commercially available formulation met the guidelines set by the British Pharmacopeia [17], whereby at least $80 \%$ of the API is released within $30 \mathrm{~min}$.
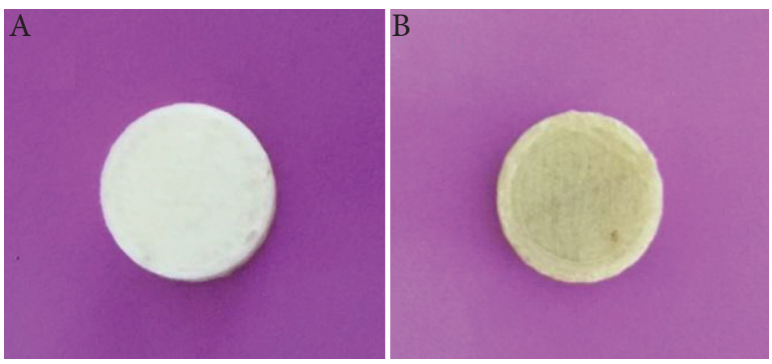

Figure $1 \mid$ Printlets (A) PVA and (B) paracetamol-loaded PVA. 


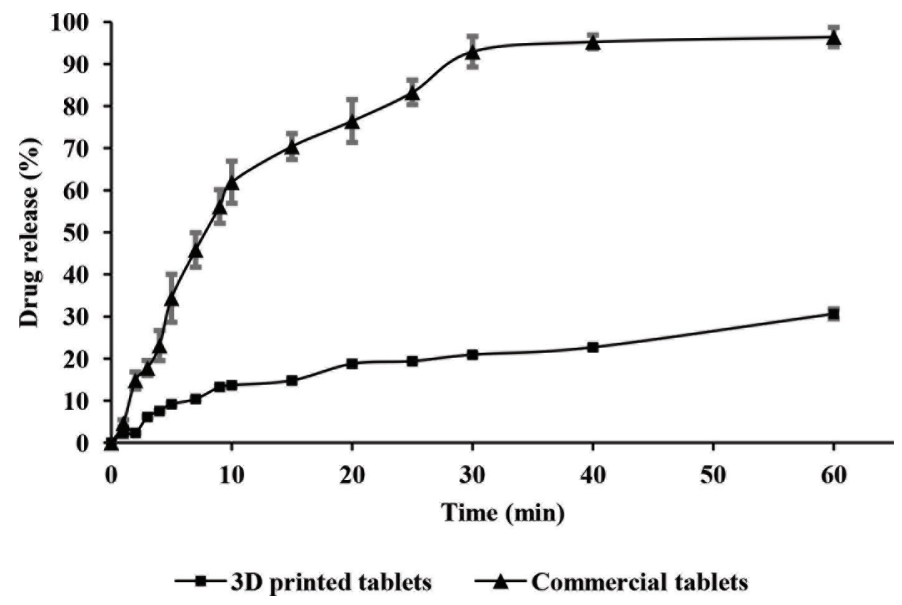

Figure 2 Dissolution profiles for commercial $(\mathbf{\Delta})$ and PVA-loaded 3D printed $(\cdot)$ tablets $(n=3$, Mean $\pm \mathrm{SD})$.

In comparison, the dissolution profile presented for the PVAloaded 3D printed paracetamol tablets did not comply with the aforementioned guidelines since only $20.91 \pm 0.32 \%$ of drug content was released from the formulation within $30 \mathrm{~min}$ and $30.61 \pm 1.18 \%$ upon experimental completion (Figure 2 ). In light of these results, further investigations were conducted to ascertain a more accurate paracetamol release profile. Consequently, PVAloaded 3D printed paracetamol tablets were left in dissolution testing conditions for a total period of $24 \mathrm{~h}$ with results showing an extended release profile of the drug (63\% drug release). Chai et al. [18] also demonstrated a similar trend in drug release rates for commercial and 3D printed domperidone tablets, where $3 \%$ of the API was released from the $3 \mathrm{D}$ printed formulation after $15 \mathrm{~min}$, with a sustained release profile emerging after $12 \mathrm{~h}$, although the drug loading was significantly lower.

\section{CONCLUSION}

Here we successfully demonstrate the 3DP of paracetamol tablets using PVA-loaded filament with a 100\% infill density. The dissolution studies of the API from 3D printed paracetamol tablets showed a sustained release profile in comparison to commercial paracetamol tablets. This work exhibits the potential FDM printing brings to the manufacture of medicines, however high extrusion temperatures for PVA printing can be seen as a possible disadvantage and the use of alternative polymers may permit printing at lower temperatures to avoid degradation of the drug. Future studies will involve the potential use of different polymers and APIs with maintained biocompatibility and expected extend release profiles, respectively.

\section{CONFLICTS OF INTEREST}

The authors declare they have no conflicts of interest.

\section{AUTHORS' CONTRIBUTION}

Conceptualization, BCS and BP; methodology, MA; analysis, MA, BCS and BP; writing - original draft preparation, BCS and BP; writing - review and editing, BCS and BP; visualization, BCS and $\mathrm{BP}$; supervision, BCS and BP.

\section{FUNDING}

We thank London Metropolitan University for funding.

\section{SUPPLEMENTARY MATERIALS}

Supplementary data related to this article can be found at https:// doi.org/10.2991/mathi.k.200829.001.

\section{REFERENCES}

[1] Prendergast ME, Burdick JA. Recent advances in enabling technologies in 3D printing for precision medicine. Adv Mater 2020;32:1902516.

[2] Shaqour B, Samaro A, Verleije B, Beyers K, Vervaet C, Cos P. Production of drug delivery systems using fused filament fabrication: a systematic review. Pharmaceutics 2020;12:517.

[3] Hsiao WK, Lorber B, Reitsamer H, Khinast J. 3D printing of oral drugs: a new reality or hype? Expert Opin Drug Deliv 2018;15:1-4.

[4] Abu Fara D, Dadou SM, Rashid I, Al-Obeidi R, Antonijevic MD, Chowdhry BZ, et al. A direct compression matrix made from xanthan gum and low molecular weight chitosan designed to improve compressibility in controlled release tablets. Pharmaceutics 2019;11:603.

[5] Garg N, Pandey P, Kaushik D, Dureja H. Development of novel multifunction directly compressible co-processed excipient by melt granulation technique. Int J Pharm Investig 2015;5: 266-74.

[6] Khaled SA, Alexander MR, Wildman RD, Wallace MJ, Sharpe S, Yoo J, et al. 3D extrusion printing of high drug loading immediate release paracetamol tablets. Int J Pharm 2018;538: 223-30.

[7] Goyanes A, Kobayashi M, Martínez-Pacheco R, Gaisford S, Basit AW. Fused-filament 3D printing of drug products: microstructure analysis and drug release characteristics of PVA-based caplets. Int J Pharm 2016;514:290-5.

[8] Goyanes A, Fina F, Martorana A, Sedough D, Gaisford S, Basit AW. Development of modified release 3D printed tablets (printlets) with pharmaceutical excipients using additive manufacturing. Int J Pharm 2017;527:21-30.

[9] Goyanes A, Buanz ABM, Hatton GB, Gaisford S, Basit AW. 3D printing of modified-release aminosalicylate (4-ASA and 5-ASA) tablets. Eur J Pharm Biopharm 2015;89:157-62.

[10] Chew SL, Modica de Mohac L, Tolulope Raimi-Abraham B. 3D-printed solid dispersion drug products. Pharmaceutics 2019;11:672.

[11] Tan DK, Maniruzzaman M, Nokhodchi A. Advanced pharmaceutical applications of hot-melt extrusion coupled with fused deposition modelling (FDM) 3D printing for personalised drug delivery. Pharmaceutics 2018;10:203.

[12] Spizzirri UG. Functional polymers for controlled drug release. Pharmaceutics 2020;12:135.

[13] Kadajji VG, Betageri GV. Water soluble polymers for pharmaceutical applications. Polymers 2011;3:1972-2009.

[14] Wei C, Solanki NG, Vasoya JM, Shah AV, Serajuddin ATM. Development of 3D printed tablets by fused deposition modeling using polyvinyl alcohol as polymeric matrix for rapid drug release. J Pharm Sci 2020;109:1558-72. 
[15] Censi R, Gigliobianco MR, Casadidio C, Di Martino P. Hot melt extrusion: highlighting physicochemical factors to be investigated while designing and optimizing a hot melt extrusion process. Pharmaceutics 2018;10:89.

[16] Erokhin KS, Gordeev EG, Ananikov VP. Revealing interactions of layered polymeric materials at solid-liquid interface for building solvent compatibility charts for 3D printing applications. Sci Rep 2019;9:20177.
[17] British Pharmacopoeia Commission. British Pharmacopoeia 2016. London: TSO; 2016.

[18] Chai X, Chai H, Wang X, Yang J, Li J, Zhao Y, et al. Fused deposition modeling (FDM) 3D printed tablets for intragastric floating delivery of domperidone. Sci Rep 2017;7:2829. 